\title{
The "Abdominal Brain"-The Wonders of Gut Feeling \& Healing \& Utilizing the 5 Elements Map of Emotions in the Small Intestine
}

\section{Livni 0*}

Pacific College of Oriental Medicine, USA

*Corresponding author: Osnat Livni, Certified Instructor, Pacific College of Oriental Medicine, USA, Tel: 6192007890; Email: osi@soulgate.net

\section{Perspective}

Volume 3 Issue 1

Received Date: January 19, 2019

Published Date: February 05, 2019

DOI: $10.23880 /$ jonam-16000166

\section{Abstract}

Our Gut has its own mind * The Intestines plays a significant role in our Physical, Emotional and Mental health. We may use the wisdom of the Gut to provide healing \& overall Well Being. * Chinese medicine names the Gut "The Abdominal Brain" and refers to it as a Thinking \& Feeling entity in the human body. * The Small Intestine embodies an inner mechanism of knowing. It isolates constructive nutrient \& supportive emotions, from distractive toxins \& un-supportive emotions. * Modern scientists are now confirming the ancient knowledge of TCM. They state that the root of the mysterious "Gut" sensations is in a network of neurons lining our alimentary canal (Gut). They call it "The second Brain" and refer to the Enteric Nervous System (ENS). * Michael Gershon explains that the ENS is a unique part of the Autonomic nerves system along with the Sympathetic and parasympathetic divisions. * Both the Cranial brain and the abdominal brain originate during fetal development from tissues called the Neural Crest. They are connected by a group of nerve fibers called the Vagus (also vagal) nerve. * The two brains collaborate with each other providing a full spectrum of survival wisdom. Cranial brain contributes Intellectual wisdom \& mental intelligence. The Abdominal brain offers Instinctive wisdom \& Emotional intelligence. * The "Shen" of the Heart- similar to the Vagus nerve - forms a connection between the Thinking brain and the Instinctive brain. * The meridian of the S.I creates another interaction between the head and the Gut as it coordinates the activity of the Jaw with the Ileocecal valve. * According to the Healing Tao, stress and blocked emotions form physical knots in the Small Intestine. Their location follows the five elements wisdom of Chinese medicine in relations to the emotional energy of the Internal Organs. *We can utilize this 5-element map for both, Evaluation as well as Treatment of Digestive disorder and emotional imbalances. *The Taoist Internal Organ Massage provides healing of Body (Gut), Mind (Brain) \& Spirit (Shen). These methods rejuvenate the entire body, Restoring overall vitality, creativity and wellbeing.

Keywords: Abdominal Brain; Feeling \& Healing; Absorbing Nutrients; Small Intestine 


\section{Journal of Natural \& Ayurvedic Medicine}

\section{Introduction}

Have you ever felt "Gut Feeling Intuition", or a "Second thought", or "Butterflies in your Belly? " These are familiar expressions of common, yet perplexing, phenomena many people share. "Vacation G.I disorder" is another common and puzzling condition. It makes sense when people experience abdominal discomfort and Irritable Bowel Syndrome as a chronic condition when under stress, as a response to medication, or during illness. But why would they develop such stagnation when their Body \& Mind is healthy and fully relaxed?

As one of my clients complained:
"I don't get it... I'm finally on vacation, I'm relaxed as can be. So why do I get so blocked? What's going on with my Gut? It's supposed to work better when I'm relaxed, but it doesn't... as if it has a mind of its own..."

Well... It actually does! Our Gut has a mind of its own. A different one then the thinking mind. It affiliates with the presence of feelings \& somatic response to emotional experiences. We are often not aware that an emotional experience is taking place, and yet the Gut knows. It then reacts by expressing various bodily sensations and disorders. The Taoist and ancient Chinese healers associate these peculiar occurrences with the activity of the Small Intestine/Gut. They named this organ "The Abdominal Brain".

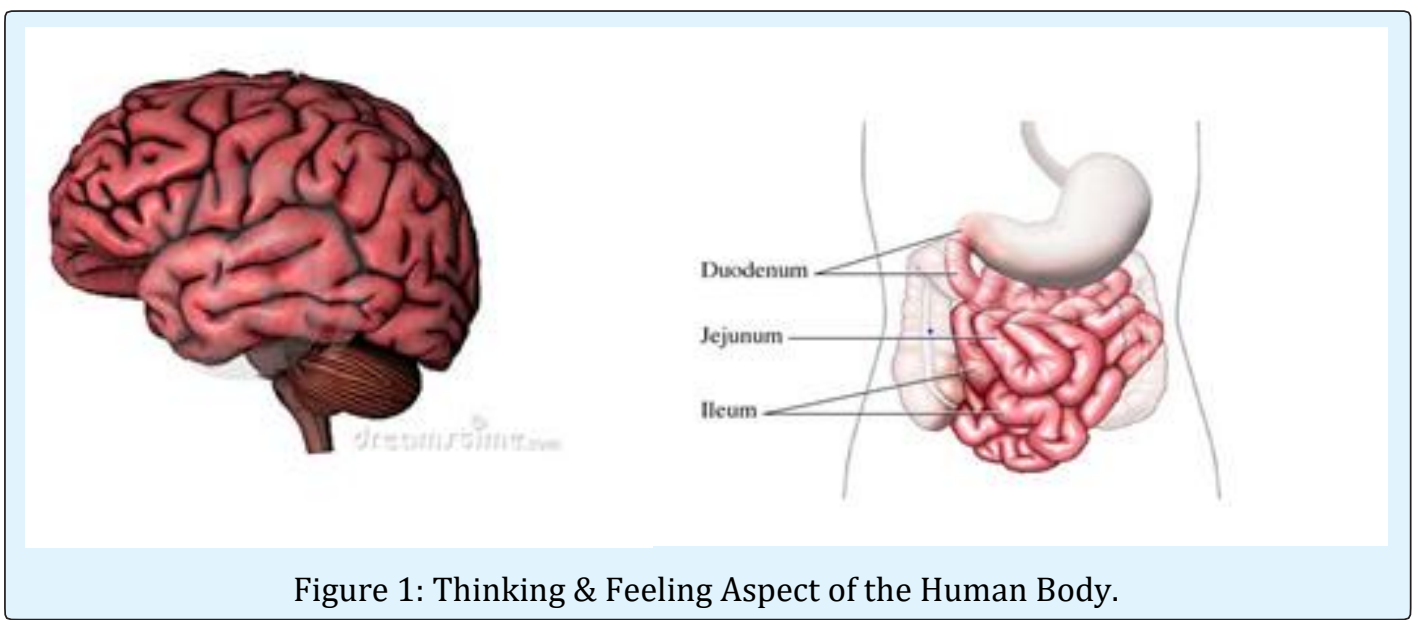

\section{The Small Intestine \& the Brain in TCM \& Modern Medicine}

Modern scientists explain that both, the cranial brain and the abdominal brain originate prenatally from the same tissues called the Neural Crest. They share a visual similarity as well as significant mutual functions.

The Intestines/ Gut play an important role in our Physical, Emotional and Mental health:

- The Small Intestine embodies an inner mechanism of knowing.

- The Large Intestine conducts the process of letting go.

- We may use the wisdom of the Gut to provide healing \& overall Well Being.

According to the wisdom of Chinese medicine, the internal Organs are containers of our Essence-our physical, emotional and spiritual life force. Each organ holds a portion of the body's physical energy. It performs multi-biological tasks in order to maintain life. Each organ also contains a portion of the Emotional \& Spiritual vibrations. Therefore, it fulfills emotional functions, cultivating the quality of our inner life. Chinese Medicine describes the main role of the Small Intestine as: "Separating the pure from the impure." This is a slightly different view from Western science, which defines the main job of the Small Intestine as "Absorbing nutrients."

Off course, TCM also includes the absorption of nutrients as a very important task. Yet, it focuses on the complex process that is taking place in the Gut prior to absorption. This is the process in which the Small Intestine isolates the toxins from the nutrients, making sure only pure nourishment will penetrate into the blood stream, and toxins will be eliminated out of the body via the large intestine. 


\section{Journal of Natural \& Ayurvedic Medicine}

\section{The Small Intestine Embodies an Inner Mechanism of Knowing}

This organ can discriminate what part of the food is beneficial for the body and what part is harmful. On some level, the Small Intestine actually makes decisions. It knows the difference between what is constructive to us and what is destructive.

As a container of $\mathrm{Qi}$, the Small Intestine performs its tasks on all levels - Physically as well as Emotionally and Spiritually. It "digests" life's experiences as it does with food. It decides what part of the occurrence should be assimilated and utilized as wisdom and what part should be eliminated as waste. This process is very similar to the thinking action of the Cranial Brain.

\section{The SI Contains the space of Wisdom in our Body}

Inspired by the Serenity prayer of the 12 steps; the key to inner peace and well-being is "The Wisdom to know the Difference" between the supportive components of life and the un-supportive one; When to take action and when to practice acceptance. The Small Intestine isolates constructive nutrient \& supportive emotions, from distractive toxins \& unsupportive emotions. It literally "Knows the difference" in what is good for us and what is not. Therefore, it holds space for our wisdom and "Gut feeling intuition".

\section{Modern Science is Now Confirming the Ancient Knowledge of TCM}

Recent research provides anatomical \& chemical interpretations to the energetic concepts of Chinese medicine. As well as to the mysterious "Gut feeling" responds many people experience. Scientists state that the root of eccentric "Gut" sensations is in a network of neurons lining our alimentary canal known as Gut. It is such a complex and extensive system that some scientists have nicknamed it the "Second Brain." They refer to the Enteric Nervous System (ENS), which is an independent nervous system within the intestinal walls. Michael Gershon, is an expert in the growing field of NeuroGastroenterology. He is the author of the book "The Second Brain." (Michael Gershon is the chairman of the Department of Anatomy and Cell Biology at New York Presbyterian Hospital/Columbia University Medical Center).

In his book, Michael Gershon explains that the Enteric Nervous System (ENS) is like a second brain. It is a unique part of the Autonomic nerves system, along with the sympathetic and parasympathetic divisions. The ENS consists of neurons embedded in the walls of our Gut. The second brain contains about 100 million neurons, more than in either the spinal cord or the Peripheral Nervous System. This multitude of neurons in the Enteric Nervous System enables us to "feel" the inner space of our gut, its activity and contents.

Different then the Sympathetic and parasympathetic systems, the Enteric Nervous System (ENS) of the Gut works independently of the Central Nervous System (CNS) of the brain and spinal cord. It has its "own brain". Yet, there is a constant communication between the cranial brain and these three components of the nervous system.

\section{The Interaction between the Cranial Brain and the Abdominal Brain}

There is a constant communication between the cranial brain and the various components of the Autonomic Nervous System. Both brains originate during fetal development from tissues called the Neural Crest. These tissues divided to form the two brains and are connected by a group of nerve fibers called the Vagus (also vagal) nerve. The Brain-Gut connection through the Vagus nerve creates a complex relationship between the Central Nervous System (CNS) and the Enteric Nervous System (ENS). They are communicating with one another as the Vagus nerve sends a steady stream of messages between the cranial brain and the abdominal brain.

\section{The Two Brains Collaborate with Each Other Providing a Full Spectrum of Survival Wisdom}

- The cranial brain contributes an intellectual wisdom of Logic, Reason \& acquired Knowledge, which comes from our mental intelligence, as well as our understanding of life's experiences.

- The Abdominal brain offers the natural organic wisdom of Instincts, Intuition \& Inner Guidance, which generates from our emotional intelligence, as well as our authentic connection to the ever-present life force in the body.

Together they form a very important mechanism for survival. When the Small Intestine detects a harmful substance in the food we insert, it immediately sends survival warning signs to the Cranial brain, so together they may fight off and expel the invasion. A similar process is taking place emotionally. When the Gut identifies a harmful situation, it starts transmitting 


\section{Journal of Natural \& Ayurvedic Medicine}

alarming messages to the brain, calling attention to the perceived risk.

\section{The "Shen" Forms a Connection between the Thinking Brain and the Instinctive Brain}

Chinese Medicine does not mention the two nervous systems and their connection through the Vagus nerve. Yet, TCM suggests a parallel concept by looking at the link between the Small Intestine and the Heart, as Yin \& Yang pair organs of the Fire element. The Small intestine embodies the Abdominal Brain, and the Heart houses the "Shen"="spirit". This subtle ethereal aspect of our being governs our mind, consciousness, emotions, thoughts, creativity \& memory. It also rules the state of relaxation during sleep. It, therefore, allows the life-giving systems of the body to fulfill their tasks. The Shen-like the Vagus nerve-forms a connection between the Thinking brain and the Instinctive brain. It relaxes the cranial brain and the CNS, allowing the abdominal brain and the ENS to govern the functions of the internal organs in maintaining life. The function of the Shen in providing relaxation and restful sleep is fundamental to our health and wellbeing. Modern medicine describes this concept as "Rest \& Digest."

\section{The Meridian of the Small Intestine}

It creates another interesting bond between the head and the Gut. This meridian begins in the depth of the abdomen. It generates from the small Intestine, flows superficially through the exterior portion of the arm and ends at the Jaw. It provides an important link between the Jaw and the Small Intestine. The act of chewing initiates the activity of the Ileocecal valve. As the valve opens, digested content moves from the Small Intestine into the Large Intestine, clearing space for the new meal to enter the gut.

\section{Stress and Tension}

It plays significant roles in many digestive disharmonies \& Illnesses. Stressful mind prevents restful sleep. Chinese medicine refers to sleep disorders as "Disturbed Shen" which interferes with the functions of the organs and the abdominal brain.

\section{Tension is Often a Physical Expression of Emotional Stress}

It blocks the circulation and constrains various muscles in the body, including the Jaw and the muscular tissues of the organs. TMJ tightness and grinding teeth habit may interfere with the natural rhythm of the Ileocecal valve, as well as the Qi flow in the Small Intestine meridian and organ. Stress disturbs the delicate interaction among the various divisions of the nervous system, as well as the communication between the Cranial and the abdominal brains. That creates physical \& emotional obstructions.

\section{Emotional Impact on the Abdominal brain}

The Emotions are the bridge between the subtle energy of the body and the denser vibrations of the physical tissues.

\section{Emotions have Two Components}

- $\quad$ The Energetic component of E- Motion = Energy in Motion. The flowing waves of subtle vibrations expressing the electro-Magnetic field of Qi.

- The physical component of Feelings. As the refine energy of the E-motions accumulates more density, it produces chemical changes and physical Sensations.

When our emotional energy flows freely, we enjoy the pleasure of circulating Qi. We experience supportive feelings of wellbeing, a sense of openness, vitality, creativity and balance. When we are unable to fully process and resolve stressful situations, the "un-digested" emotional impact may stay in the Gut, forming blocked emotions. Like the experience of an un-digested food, the Gut may become overwhelmed. Stagnation and physical toxins may build up causing chemical conversions as well as contractions in the muscular tissue of the intestinal walls. The Small Intestine - as a container of Qi - will hold blocked emotional vibrations in its energetic layers. It would wait for another chance to surface them in order to "Re- digest," process and transform the stagnant emotions. The experience of physical and emotional discomfort is often called "Negative" or "Unsupportive" emotions. Blocked Emotions are often the expression of Survival Tension The Abdominal Brain - as the Autonomic nervous system-is in charge on survival "fightflight" response. The abdominal brain may perceive a survival risk when external circumstances present a true survival danger, as well as when past emotional blocks get triggered and surface to awareness.

Survival tension from, either a current experience, or a past one, may cause agitation in the small intestine. The abdominal brain attempts to connect with the cranial brain and call our attention to the perceived danger. The alarming signals may produce numerous symptoms such as buzzing "butterflies" and other peculiar sensations and 


\section{Journal of Natural \& Ayurvedic Medicine}

chemical changes. These could lead to even more severe syndromes of tightness, inflammation, pain, nausea, vomiting, diarrhea, constipation and Irritable bowel syndrome.

This may explain why many travelers may think in their cranial brain that they are relaxed while enjoying their vacation. Yet in the unfamiliar environment, the change of routine and activities may trigger survival tension in their Abdominal Brain. Also, sometimes when we allow relaxation to take place, the blocked emotions from the past re-surface. They call for resolution by producing alarming signals such as tightness, knots, and some of the above symptoms.

Conscious and/or unconscious stress may create discrepancy in the communication between the two brains. That causes an additional tension which may lead to constant constriction in the intestine, disturbances of the peristaltic flow and may produce chronic conditions in the digestive \& elimination systems.

\section{The Five Elements Emotional Map of the Abdominal Brain}

According to the Healing Tao, there is a system in which stress and blocked emotions form specific knots and tangles in the Small Intestine.

\section{Knots}

They are in the surface layers of the body. They appear as thickened lumpy flesh, which affects the fascia, lymph nodes, superficial nerves and muscles.

\section{Tangles}

They are found in deeper areas affecting nerves, blood vessels and connective tissues. As they become twisted together fatty tissue \& lymph node may get stuck inside. Both are the cause of toxicity, contractions and spasm in certain regions of the abdominal brain. The locations of these blocks correspond to specific "Un-digested" emotions. This method follows the Five Elements wisdom of Chinese medicine in relations to the emotional energy of the Internal Organs:

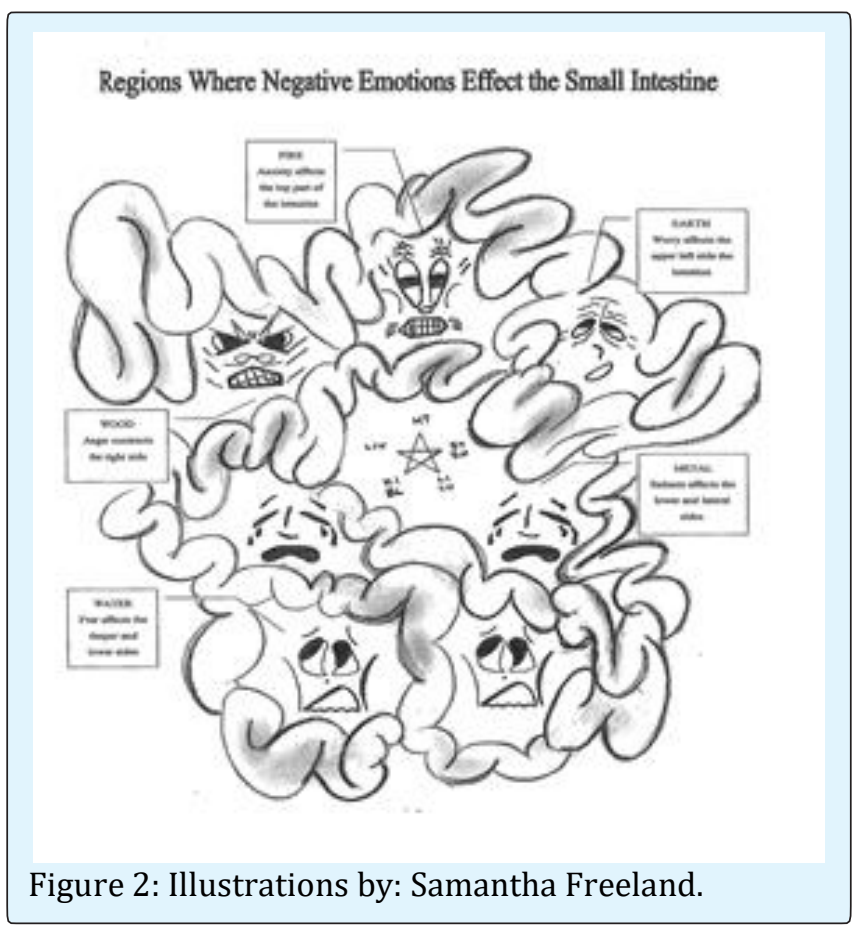

\section{The Five Elements Emotional Map of the Abdominal Brain \\ Wood-Anger}

Contracts the upper right side of the intestine, in the area close to the Liver \& Gall Bladder. Opening this region helps transforming Anger to: Forgiveness \& Compassion.

\section{Fire - Anxiety \& Depression}

They affect the upper part of the intestine - in the area of the Heart.

Opening this area helps transforming anxiety to: Excitement, Joy, Love \& Gratitude.

\section{Earth-Worry}

Tightens the upper left side of the intestine-in the area of the Stomach \& spleen. Opening this part helps transforming worries to: Fulfillment, Contentment \& Calmness. 


\section{Journal of Natural \& Ayurvedic Medicine}

\section{A Sense of Balance, Harmony, Abundance, Prosperity and Clarity of our Spiritual Path}

\section{Metal - Grief \& Sadness}

It blocks the lower left part of the intestine-in the area of the descending colon of the Large Intestine. Opening this region helps transforming grief to: Happiness \& Courage to let go and flow with life's changes.

\section{Water- Fears}

It contracts the lower part and side portion of the intestine - the area of the Kidneys, Bladder and Reproductive system. Opening these locations helps transforming fears to: Inner peace, confidence \& Trust. We can utilize the five elements emotional map of the Abdominal Brain for both, Evaluation as well as Treatment of Digestive disorders and emotional stress. The Taoist Internal Organ Massage provides healing of Body, Mind \& Spirit. It offers powerful tools to restore the organic connection among the Abdominal brain of the Small Intestine (Body), the nervous systems of the Cranial brain (Mind) and the Shen of the heart (Spirit). Unique relaxing manipulations of the Abdomen, the Ileocecal valve as well as the Jaw, are applied in order to open up physical \& emotional blocks; release stress \& toxins from the organ; improve the natural flow of communication between the two brains; harmonize the interactions of the Sympathetic, Parasympathetic \& Central nerves systems. These healing methods rejuvenate the entire body, Restoring overall vitality, creativity and wellness.

\section{Summary}

\section{Our Gut has its Own Mind}

1. The Intestines plays a significant role in our Physical, Emotional and Mental health. We may use the wisdom of the Gut to provide healing \& overall Well Being.

2. Chinese medicine names the Gut "The Abdominal Brain" and refers to it as a Thinking \& Feeling entity in the human body.

3. The Small Intestine embodies an inner mechanism of knowing.

4. It isolates constructive nutrient \& supportive emotions, from distractive toxins \& unsupportive emotions.
5. Modern scientists are now confirming the ancient knowledge of TCM. They state that the root of the mysterious "Gut" sensations is in a network of neurons lining our alimentary canal (Gut). They call it "The second Brain" and refer to the Enteric Nervous System (ENS).

6. Michael Gershon explains that the ENS is a unique part of the Autonomic nerves system along with the Sympathetic and parasympathetic divisions.

7. Both the Cranial brain and the abdominal brain originate during fetal development from tissues called the Neural Crest. They are connected by a group of nerve fibers called the Vagus (also vagal) nerve.

8. The two brains collaborate with each other providing a full spectrum of survival wisdom. Cranial brain contributes Intellectual wisdom \& mental intelligence. The Abdominal brain offers Instinctive wisdom \& Emotional intelligence.

9. The "Shen" of the Heart - similar to the Vagus nerve forms a connection between the Thinking brain and the Instinctive brain.

10. The meridian of the S.I creates another interaction between the head and the Gut as it coordinates the activity of the Jaw with the Ileocecal valve.

11. According to the Healing Tao, stress and blocked emotions form physical knots in the Small Intestine. Their location follows the five elements wisdom of Chinese medicine in relations to the emotional energy of the Internal Organs.

12. We can utilize this 5-element map for both, Evaluation as well as Treatment of Digestive disorder and emotional imbalances.

13. The Taoist Internal Organ Massage provides healing of Body (Gut), Mind (Brain) \& Spirit (Shen). These methods rejuvenate the entire body, Restoring overall vitality, creativity and wellbeing.

\section{References}

1. Gershon M (2019) The Second Brain. Harper Perennial, pp: 336.

2. Mantak Chia (2001) Chi Nei Tsang, USA. 